\title{
Multi-outlet network load balancing solution
}

\author{
Liang Wang \\ Shanghai Urban Management College Shanghai China,200232 \\ civilwon@hotmail.com
}

Keywords: Multi-outlet network access load balancing

\begin{abstract}
Due to the current number of domestic Internet access provider (ISP), backbone operators and distributed in different regions, which leads to a different ISP access speed between slow. Meanwhile, various colleges and universities have to adapt to this network access network access environment, and use of simultaneous access Cernet and ChinaNet and other ways to enhance the campus network users of the cernet and public network access speed and can reduce the cost of network bandwidth leased. Because different ISP's network access visits is slow, so, this article from campus network flow control how the device through the campus network configuration management, DNS load balancing settings, policy routing configuration settings and other aspects to achieve improved network access speed, allowing users to automatically access the different needs of different networks in order to optimize network so greatly enhance the efficiency of network access.
\end{abstract}

\section{Introduction}

As the domestic ISP's network link is slow, generally within the university, there will be two kinds or more network access to the network center or computing center, which includes Cernet, China Net, China Mobile Net, China Unicom Net other access methods. Because Cernet development started late, insufficient bandwidth resources, access and use of higher cost, equipment redundancy and other factors, leading to its use is often inefficient but also prone to failure, for the security and stability of the campus network using a certain affected. Also, use Cernet visit China Net, and other ISP's speed is relatively slow, and for providing free access to its list of addresses outside the site may need to pay additional access fees. Therefore, in order to improve the stability of the campus network and improve network access speed and reduce network costs, often also access other ISP, such as China Net, China Mobile Net exports such as the network, which can be accessed only on a large number of Cernet educational resources and access to information resources on the Internet generally.

Because of different ISP links visits and interoperability issues, leading to a resource within the same ISP visits faster, when the cross-ISP access, the access speed is slower. As a dual-link access mode, you can avoid a single point of a link that points failure caused by paralysis of the network. When a network problem occurs, the device will automatically switch to another on a network link, in order to protect the normal network access. This is through the flow control device configuration management, DNS load balancing settings, policy routing and other aspects of dual-link network management and optimization to ensure the safe use of network load balancing.

\section{Load balancing device management}

\subsection{Hardware Optimization Management}

By adding the core layer of campus network flow control and other network devices, you can optimize access to the network, making the business systems greatly enhance the speed of access to the network.

Provides hardware flow control based on application layer optimization techniques are widely used for various types of servers, OA system, CRM, database, EMAIL, file sharing, desktop virtualization applications and a variety of industry-specific software. By providing the appropriate application protocol optimization technology that dramatically improves the application in WAN 
application efficiency. Some hardware flow control feature library by establishing protocols to control the response network application layer traffic management and through dynamic "Protocol Signature Description Language"-(PSDL) to analyze management data signature, in order to enhance the recognition accuracy of the data.

In the actual network area, management through a flexible policy configuration on the device to change the network access. Policy configuration in the configuration device or a corresponding device management objects when they can set up network access methods. In the dual-link access mode, the device automatically configures do respond.

When the network link is a normal dual connection, set the corresponding data response corresponding network link, that is, when the user needs to access the data in Cernet through the flow control policy routing, the user can automatically link to communicate via Cernet; same reason, when a user needs to access ChinaNet network data, the user's links will automatically communicate through ChinaNet. When one link fails, the device can automatically switch all network access through the normal link to communicate. Therefore, the rate of equipment control, bandwidth reservation, protocol control, and the number of concurrent control flow configuration management fine after single-link communication will greatly ease the communication network in order to achieve the campus network dual link of load balancing, as shown in Figure 1.

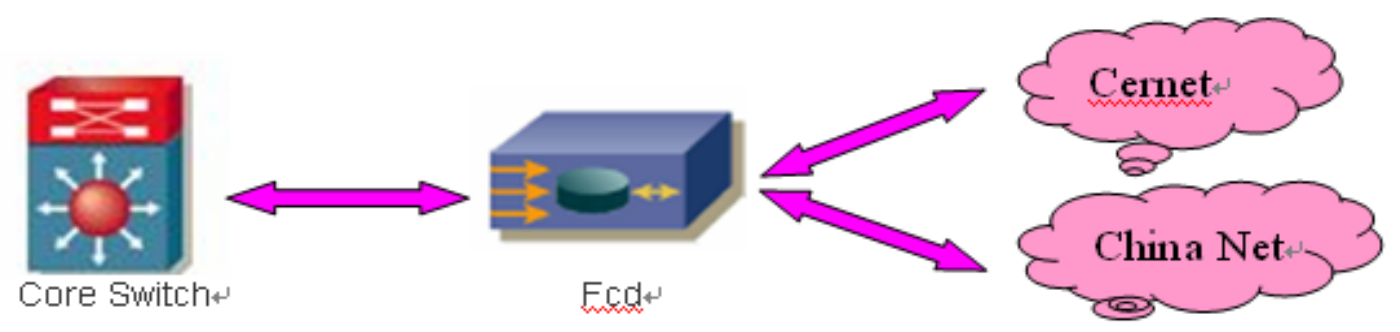

Figure 1

\subsection{Intelligent DNS resolution}

Intelligent DNS is the network access in a multi-state link IP and domain name resolution services for intelligent, you can automatically determine the visitor's IP address and resolve the corresponding IP address, that can make Cernet user can access the data can be accessed directly to Cernet server. Other users can automatically determine the ISP access automatic access to your ISP's server, so as to enhance the network access speed.

When users access the network, in the application to access the host domain name, DNS is required to access the smart server can resolve the name into its corresponding IP address. Depending on your ISP provides different network addresses, network servers were parsed into the other ISP's mapped address, and thus determine the user's network and get different DNS results.

Currently, more common intelligent DNS build by the BIND. On Linux and other open source operating system, in BIND9 environment, use View allows each source address in a different network segment in the query need different DNS, the DNS query with the appropriate information, through simple configuration can be achieved in different campus network link connect different ISP's address, in order to achieve network load balancing. Common DNS brief configuration is as follows:

Named. Conf

Include "jkw. conf"

\#jkwdz. conf

View “view-jkwdz"

match-clients $\{\mathrm{jkydz} ;$;

include "master / jkwdz. def"'

\#cemetldef jkw. txt

View "view-any" 
match-clients $\{$ any; $\}$ :

include “master / ydwl. def”

\#ydwl. Def ydwl. Txt in jkw.conf, “jkw” \{202.120.224.0. / 16; 202.120.255.0 / 14; \};

\section{Linkage of the router and firewall policy routing}

Links in the network, in order to solve a single slow Internet links, cross-ISP access when the network is slow or even disconnected, etc., can be configured on the router through different access routes, and network security and firewall configuration linkage strategy configuration can achieve network load balancing purposes.

3.1 In order to achieve a dynamic network load balancing and firewall in the router to do the following configuration, configure default routing strategy:

config route static

edit route1

set device "dv1"

set dst 0.0.0.0 0.0.0.0

set gateway 202.120.224.0

edit router2

set device "dv2"

set dst 0.0.0.0 0.0.0.0

set gateway 211.136.17.107

\subsection{Configuring PBR}

By PBR, you can achieve different network access access different networks. Configuration commands are as follows:

config router policy

edit route1

set input-device "porta”

set src 0.0.0.0 0.0.0.0

set dst 202.120.224.0 255.254.0.0

set output-device “portb”

edit router2

set input-device " porta”

set src 0.0.0.0 0.0.0.0

set dst 202.120.255.0 255. 252. 0. 0

set output-device " portb”

edit router10

set input-device "porta"

set src 0.0.0.0 0.0.0.0

set dst 0.0.0.0 0.0.0.0

set output-device "portc"

set gateway 211.136.17.107

\subsection{Linkage of the router and firewall}

Configure the network firewall and routers do linkage, set the appropriate firewall policy, when the link is disconnected, the data will automatically determine the link error, and then through another link for data communication. Policy on the firewall must be configured to set the flow of data from the porta applied to portb and porta pointing to data streaming applications pointing to portc, which makes routers and firewalls can generate linkage of the effects, get more exports of network load balancing effect. 


\section{Summary}

In the current network access environment, different ISP access on campus to assume a different role, to achieve different ISP access network between each other, but also to enhance the process of accessing the access speed of the network has become routine management a top priority. By using the campus network flow control device configuration management, DNS load balancing settings, policy routing settings for the actual operation of the network, dynamically allocating and adjustment, can be a quick route through the best way to choose the best access path, making speed network access to each other to achieve the multi-link network load balancing.

\section{References:}

[1] Policy routing technology in a multi-outlet Campus Network Application <Computer and Information Technology> Liu Hai tao, Huang Jia lin 2012

[2] Multi-link access and flow control applications in the campus network < Network Security Technology \& Application> Wang Guan yu 2012

[3] Policy-based routing construction with double outlet campus network load balancing $<$ Technology for development> 2011

[4] Campus network bidirectional multi-link load balancing strategy analysis < Science \& Technology Association Forum> Song Xiao hui 2011

[5] Double stack link access network outlet flow optimization study <Computer Science $>$ Wang Yu jie Wang Bin xin 2012

[6] Double outlet campus network router implementations < Wuhan Metallurgical Management Institute $>$ Qian Jianggong 